\title{
Tuberculous pericardial disease: a focused update on diagnosis, therapy and prevention of complications
}

\author{
Kishendree Naicker, Mpiko Ntsekhe \\ Division of Cardiology, Department of Medicine, University of Cape Town, Groote Schuur Hospital, Cape Town, South Africa \\ Contributions: (I) Conception and design: All authors; (II) Administrative support: All authors; (III) Provision of study material or patients: All authors; \\ (IV) Collection and assembly of data: All authors; (V) Data analysis and interpretation: All authors; (VI) Manuscript writing: All authors; (VII) Final \\ approval of manuscript: All authors. \\ Correspondence to: Mpiko Ntsekhe, MD, PhD, FACC. The Cardiac Clinic, New Groote Schuur Hospital, University of Cape Town, Cape Town, \\ South Africa. Email: mpiko.ntsekhe@uct.ac.za.
}

\begin{abstract}
Tuberculous pericarditis (TBP) is the most important manifestation of tuberculous heart disease and is still associated with a significant morbidity and mortality in TB endemic areas. The high prevalence of the disorder over the last 3 decades has been fueled by the human immunodeficiency virus/AIDS (HIV/ AIDS) pandemic in these areas. The objective of this review is to provide a focused update on developments in the diagnosis and therapy of this condition, prevention of its complications, as well as future novel therapies. The definitive diagnosis of a tuberculous etiology in patients with suspected TBP continues to pose a challenge for clinicians. Clinical prediction scores, although never formally validated have been used with some success. However, they may be prone to both over and underdiagnosis due to lack of pericardial fluid analysis. Recent studies evaluating Xpert MTB/RIF, suggest that this advanced polymerase chain reaction (PCR) based technology does not provide increased accuracy compared to earlier iterations. However a combined two test approach starting with Xpert MTB/RIF followed by either adenosine deaminase (ADA) or interferon gamma (IFN- $\gamma$ ) may provide for significantly enhanced specificity and sensitivity cost permitting. Pericardiocentesis remains the gold standard for managing the compressive pericardial fluid and its adverse hemodynamic sequelae. A four drug anti-TB drug regimen at standard doses and duration is recommended. However recent evidence suggests that these drugs penetrate the pericardium very poorly potentially explaining the high mortality observed particularly in those who are culture positive with a high bacillary load. Constrictive pericarditis is the main long-term complication of TBP and is still a significant cause of heart failure in Sub-Saharan Africa. This is important because access to definitive surgical therapy where TBP is prevalent continues to be low, highlighting the need to develop strategies or interventions to prevent fibrosis and constriction. Recent detailed advanced studies of pericardial fluid in TBP have revealed a strong profibrotic transcriptomic profile, with high amounts of pro-inflammatory cytokines and low levels of the anti-fibrotic tetrapeptide N-Acetyl-Seryl-Aspartyl-Lysyl-Proline (Ac-SDKP). These new insights may explain in part the high propensity to fibrosis associated with the condition and offer hope for the future use of targeted therapy to interrupt pathways and mediators of tissue damage and subsequent maladaptive healing and fibrosis. The value of effective pericardiocentesis in reducing these pro-inflammatory and profibrotic cytokines and peptides in an attempt to prevent pericardial constriction has yet to be established but has generated hypotheses for ongoing and future research.
\end{abstract}

Keywords: Tuberculous pericarditis (TBP); pericardial effusion; tamponade; constrictive pericarditis

Submitted Jul 03, 2019. Accepted for publication Sep 26, 2019.

doi: $10.21037 /$ cdt.2019.09.20

View this article at: http://dx.doi.org/10.21037/cdt.2019.09.20 


\section{Introduction}

Fuelled by the high prevalence of tuberculosis (TB) and human immunodeficiency virus/AIDS (HIV/AIDS), tuberculous heart disease remains a significant clinical problem in TB endemic areas (1). The most common and important manifestation of TB heart disease is tuberculous pericarditis (TBP) which continues to be associated with significant morbidity and mortality (2). Mortality rates for TBP as high as $40 \%$ have been described among anti-retroviral therapy (ART) naïve persons living with HIV/AIDS (3) and it remains an important treatable cause of heart failure in the same regions of the world. Since the first description of the clinical manifestations, complications and outcomes of the clinical entity in the $20^{\text {th }}$ century early diagnosis and effective management of both the mycobacterium and the pericardial complications were recognized as essential (4-6). It has been close to a decade since the last comprehensive reviews of the optimal diagnosis and management of tuberculous pericarditis and its complications $(2,5,7)$. Aside from increasing the access availability and use of ART in those patients with HIV, these reviews identified a number of important focused areas which would need to be better investigated if the morbidity and mortality of the condition was to be significantly reduced. These include: (I) the need to improve the rapidity and accuracy of the diagnosis of a tuberculous etiology; (II) the need to better understand the pharmacokinetics and pharmacodynamics of anti-tb drug therapy as it relates to the effectiveness of the drugs within the pericardium in patients with TB pericarditis; and (III) the need to develop strategies to reduce long-term complications of the disorder. Since those reviews the past decade has seen the publication of a significant amount of new data particularly from SubSaharan Africa (1,8-16) designed to address some of these areas. In this focused update on the diagnosis and therapy of TBP we will review all important developments in these areas, and discuss novel interventions under exploration or with potential for future use.

\section{What's new in the diagnosis of a tuberculous etiology in TBP?}

The definitive diagnosis of a tuberculous etiology of pericarditis has been a challenge since the earliest descriptions of the disorder. Prior to the HIV-era TBP was understood to be a paucibacillary condition in which the characteristic exudative inflammatory effusion and its consequences resulted from the robust immune response to previously dormant tuberculoproteins within the pericardium (2). The traditionally tried and tested methods of diagnosing TB elsewhere in the body, such as fluid and tissue microscopy, and culture have a very poor yield (9), and biopsy of the pericardium looking for granulomas is considered invasive, risky and therefore infrequently performed (7). As illustrated in the prospective Investigation of the Management of PericarditIs (IMPI) in Africa registry (17) the practical impact of these diagnostic limitations in clinical practice is that a high proportion of patients with effusive pericarditis in TB endemic areas may be treated empirically with little attempt to establish any evidence of TB (17). The decade since 2006 has seen utility and the role of clinical prediction scores (4) biochemistry $(18,19)$, and biomarker (20) based approaches, nucleic acid amplification based techniques such as polymerase chain reaction $(\mathrm{PCR})(8,9)$ and immunology based approaches, all being investigated in order to find a rapid accurate and cost effective diagnostic approach to establishing a tuberculous etiology.

\section{Clinical prediction scores}

Reuter et al. identified several variables associated with TB to determine the most widely used clinical prediction score. It assigns weighted points to symptoms of TB: weight loss1 point, night sweats -1 point and fever-2 points. Normal white cell count and serum globulins which are known to increase significantly in the setting of TB were each assigned 3 points (4). In the derivation study performed to establish the clinical prediction rule, an aggregate score of greater than 6 was found to have a sensitivity of $86 \%$ and specificity of $85 \%$ (4). While this TBP diagnostic index has never been validated formally in a prospective study, it was used successfully as an inclusion criteria for patients enrolled in the large Pan African IMPI randomized control trial of immunotherapy for TBP (14). A major limitation of the score is that because it requires no pericardial fluid analyses, it is particularly prone to mis-diagnosing patients with bacterial co-infection, malignancies and other illness characterized by constitutional symptoms (7).

\section{Biochemical and biomarker based approaches}

Adenosine deaminase (ADA) is an enzyme whose activity correlates closely with activation of subsets of T-lymphocytes involved in the immune responses to 
TB (21). Since 1995 there have been close to three dozen studies which have tested the utility of ADA in the diagnosis of TBP. A recent meta-analysis of 11 studies with close to 800 participants has been published (18), the authors reported a pooled sensitivity of 0.90 , specificity of 0.85 and negative likelihood of $15 \%$. They concluded that while the test was sufficiently accurate, given the heterogeneity and design of many of the studies the results of the ADA in individual patients needed to be interpreted in the context of the patients history and other investigations and not as a stand-alone test. Importantly the performance and accuracy of the test is not diminished in the setting of HIV and AIDS.

Interferon gamma (IFN- $\gamma$ ) is a cytokine that is produced by T-cells in response to TB antigens, and plays a pivotal role in the protective immune response to active TB. High levels of measured IFN- $\gamma$ are therefore indicative of the presence of active TB. Of the multiple studies to determine the accuracy and potential role of IFN- $\gamma$ in the diagnosis of TBP, four of them with close to 500 patients were conducted in high TB prevalence areas with a prospective design and were included in a meta-analysis. The authors concluded that the diagnostic accuracy of the tests were extremely high with sensitivities and specificities approximating 98 and 99 respectively (20). To date the test has been limited in clinical practice by the lack of availability of an easily accessible inexpensive commercial assay.

\section{Nucleic acid amplification based techniques}

The potential and promise of nucleic acid amplification techniques in the diagnosis of TBP following their almost spectacular success in the diagnosis of pulmonary and other forms of TB has not been realized to date. The early limitations of high false negative results and overly poor sensitivity have never been overcome (22). The accuracy of Xpert MTB/RIF, the new World Health Organization (WHO) endorsed, PCR rapid test which is widely used to simultaneously detect Mycobaterium tuberculosis (MTB) and Rifampicin sensitivity in sputum (23) has recently been compared to both ADA and IFN- $\gamma$ in effusive pericarditis. The sensitivity of Xpert MTB/RIF was found to be an unacceptable $53-70 \%$. In the study, IFN- $\gamma$ assays were found to be the optimal first line diagnostic tool for TBP $(10,24)$. The authors suggested that a combined two test approach starting with Xpert MTB/Rif followed by either ADA or IFN- $\gamma$ provided maximal sensitivity and specificity of $>97 \%$ (10). However the practicality and cost effectiveness of such a strategy in resource limited settings where TBP is most prevalent would be a major limitation. Whether or not the newer Xpert MTB/RIF Ultra assay developed to improve accuracy in extrapulmonary and paucibacillary disease will perform better in TBP is unknown but the improved design features and operational aspects of the new improved test are promising (25).

\section{Imaging}

Chest radiograph, electrocardiogram and echocardiography are the essential first line imaging tools for diagnosis and evaluation of TBP (1). Echocardiography remains the primary tool used for confirming the presence of a pericardial effusion and determining its size and amenability to pericardiocentesis (26). The use of multimodal imaging in pericardial disease such as computed tomography (CT), cardiac magnetic resonance imaging (MRI) and flurodeoxyglucose-positron emission tomography (FDGPET) is now supported by recent guidelines $(26,27)$. Cardiac MRI is able to better delineate the distribution and amount of pericardial fluid compared to echocardiography $(28,29)$ which supports more accurate evaluation for pericardiocentesis. Cardiac MRI may also help establish pericardial thickening, with thickening greater than $6 \mathrm{~mm}$ considered highly specific for constrictive pericarditis $(29,30)$. The use of FDG-PET imaging in pericardial disease is established and its ability in differentiating tuberculous pericarditis from idiopathic pericarditis demonstrated in a small study (31). Patients with active tuberculosis pericarditis showed more multifocal FDG uptake compared to regional uptake in those with acute idiopathic pericarditis (31). However, in resource limited settings where TBP remains most prevalent specialized imaging such as cardiac MRI and FDG-PET is often unavailable or requires long waiting periods which makes their functional utility difficult.

\section{What's new in the treatment of TBP and prevention of its complications}

\section{Anti-tuberculosis therapy}

The treatment of TBP targets three distinct but related goals: kill and or control the mycobacterial infection itself; prevent and or reduce the risk of the short-term cardiovascular effects of the inflamed pericardium; and 
prevent the long-term cardiac sequelae of maladaptive healing of the pericardium, such as fibrotic constriction. Standard four drug anti-TB drug regimens, used for pulmonary $\mathrm{TB}$ are recommended by global bodies such WHO for the treatment of TBP in patients with and without HIV (32). However, to date there have never been studies of the comparative effectiveness of different antiTB regimens with regards to dose or duration of therapy for the treatment of TBP. In a paucibacillary condition in which the death and damage to the host is mainly immune mediated and not related to bacillary load, it is possible that the consequences of suboptimal anti-TB drug regimens may not be clinically significant. However there is growing evidence that in the HIV era patients with TBP are more likely to have disseminated disease (12), with culture positive pericardial disease and that the high incidence of short- and long-term adverse outcomes is closely associated with initial bacillary load (33). This is particularly important because of recent data showing that there is poor penetration of anti-TB drugs into the pericardium (34). In a small sub-study designed to investigate anti-TB drug concentrations within the pericardium and compare them to the minimum inhibitory concentrations (MICs) of clinical MTB isolates, the authors reported that detectable drug levels were several-fold lower than the isolate MICs (34). Specifically, the non-protein bound levels of Rifampicin, Pyrazinamide and Ethambutol measured serially over the first 24 hours after receiving oral anti-TB therapy were low. In light of this information, the anti-mycobacterial effectiveness of these current drug regimens in TBP needs close re-evaluation and there may be a strong case to assess the impact and safety of higher doses on pericardial penetration, ability to reduce bacillary load and clinical outcomes in clinical trials.

\section{Management of compressive pericardial fuid}

There has been little information on new strategies to optimize the need for prompt relief of the compressive pericardial fluid that are the norm in TBP. Recommendations for evacuation of the pericardium in virtually all patients with moderate and large effusions have remained unchanged over the last decade $(26,35)$. Compared to the less than $25 \%$ of participants who underwent routine pericardiocentesis in the first Pan African IMPI registry (17), this proportion was over $60 \%$ in the 1,400 patient IMPI trial conducted 5 years later suggesting that guidelines and recommendations on this issue are filtering into clinical practice (14). Constrictive pericarditis is the main longterm complication of TBP occurring in up to $25 \%$ of patients following the diagnosis of the effusive phase of the disease (2). The incidence of constrictive pericarditis is significantly higher (31.65 per 1,000 cases) than it is for almost all other causes of pericarditis except purulent pericarditis (36). This is important because post-tuberculous constrictive pericarditis is a significant and relatively common cause of heart failure in many regions of subSaharan Africa (37) where access to and the availability of the definitive surgical therapy is low (2). Furthermore, where the operation is available it is associated with significant perioperative risk of both morbidity and mortality (15).

\section{Prevention of constrictive pericarditis}

Over the last decade much effort has been spent exploring and understanding the pathways and mechanisms for the high rates of post TB constrictive pericarditis (38). Work in this area is important for the potential discovery of targets for early interventions to prevent fibrosis and maladaptive remodelling and healing of the pericardium post TBP. Using a transcriptomic approach with paired wise analysis of the activated genetic response within the pericardium, a strongly profibrotic genetic profile was identified in the pericardial fluid of participants with TBP (39). The authors reported a transcriptomic gene expression signature of 17 genes that were associated with either regulators of fibrosis or actual fibrosis in both HIV infected and uninfected hosts. Further work to identify the downstream protein products of that gene expression would be important. The same group of investigators used ELISA to perform paired wise analysis of the cytokine profile of participants with TBP undergoing pericardiocentesis. Investigators found the serum of patients with the condition to have elevated transforming growth factor (TGF) beta and interleukin 10 (IL-10) compared to matched controls with no disease. These, cytokines are well established role players in the pathways of fibrosis throughout the body (40). Interestingly there was little TGF-beta in the pericardium which was dominated by pro-inflammatory cytokines. Of equal intrigue was the finding in a separate study that the pericardial fluid of participants with TBP had lower levels of the anti-fibrotic housekeeping tetrapeptide N-AcetylSeryl-Aspartyl-Lysyl-Proline (Ac-SDKP) compared to controls without TB (41). These findings are particularly interesting as AcSDKP is metabolized and broken down 
by angiotensin converting enzyme (ACE) suggesting a potential role for ACE-inhibitors in TBP as an intervention to keep peptide levels and anti-fibrotic activity high in order to reduce the likelihood of pericardial fibrosis $(16,41)$. Finally, the observation that in recent studies of TBP in which pericardiocentesis rates achieved were well over $60 \%$, the rates of constrictive pericarditis were considerably lower $(24,40,42)$ than historical cases series and studies in which pericardiocentesis was only performed mainly for tamponade $(17,43)$, may itself be a hypothesis worth testing formally. Namely that effective pericardiocentesis by removing pro-inflammatory and profibrotic cytokines and peptides from the pericardium may be an effective means of reducing pericardial fibrosis and subsequent pericardial constriction. To date a small number of trials and proof of concept studies of interventions to reduce or prevent the rate of constrictive pericarditis $(11,14,38)$ have been recently completed or are still active. The IMPI trial was a multicenter, factorial-design trial that randomly assigned 1,400 patients with pericarditis to receive a course of high-dose prednisolone tapered over a course of 6 weeks compared to placebo. In the trial, although the primary endpoint was not met, both HIV infected and uninfected participants randomized to receive prednisolone had a significantly lower incidence of constrictive pericarditis (14). Elsewhere, based on the observation that colchicine can prevent recurrences of acute idiopathic pericarditis (44) a group of South African investigators tested the hypothesis, that a 6-week course of the agent would reduce rates of constrictive pericarditis compared to a placebo controlled group. No evidence of benefit was demonstrated in the small underpowered study (45). Finally, a study to investigate the hypothesis that thrombolysis, by breaking up fibrin strands within the pericardium and facilitating a more complete evacuation of the pericardium may reduce rates of progression to constrictive pericarditis is underway with the pilot phase being conducted in South Africa (https://clinicaltrials.gov/ct2/show/results/ NCT02673879).

\section{Conclusions}

TBP remains a prevalent and lethal condition in SubSaharan African and other TB endemic areas of the world particularly where HIV/AIDS prevalence is high. Over the last decade there has been a considerable amount of work to enhance our understanding and advance our knowledge of this condition. In this review we have provided a focused summary on advances made using modern methods of rapidly and accurately establishing a tuberculous etiology. Although not widely available yet, IFN- $\gamma$ is the single most accurate test while ADA testing, used and interpreted in context, remains readily accessible in most laboratories and clinically useful. The promise of Xpert and other nucleic acid amplification based technologies has not been fulfilled to date. Emerging information that anti-TB drugs penetrate the pericardium poorly needs to be explored further and may offer an area to specifically focus on in order to improve the early high mortality rates associated with the condition. Finally progress in our understanding of the mechanisms and pathways of fibrosis within the pericardium may offer hope for the development of more targeted anti fibrotic therapy in the near future.

\section{Acknowledgments}

Funding: None.

\section{Footnote}

Provenance and Peer Review: This article was commissioned by the Guest Editor (Ntobeko A. B. Ntusi) for the series "Cardiovascular Diseases in Low-and Middle-Income Countries" published in Cardiovascular Diagnosis and Therapy. The article was sent for external peer review organized by the Guest Editor and the editorial office.

Conflicts of Interest: Both authors have completed the ICMJE uniform disclosure form (available at http://dx.doi. org/10.21037/cdt.2019.09.20). The series "Cardiovascular Diseases in Low- and Middle-Income Countries" was commissioned by the editorial office without any funding or sponsorship. The authors have no other conflicts of interest to declare.

Ethical Statement: The authors are accountable for all aspects of the work in ensuring that questions related to the accuracy or integrity of any part of the work are appropriately investigated and resolved.

Open Access Statement: This is an Open Access article distributed in accordance with the Creative Commons Attribution-NonCommercial-NoDerivs 4.0 International License (CC BY-NC-ND 4.0), which permits the noncommercial replication and distribution of the article with the strict proviso that no changes or edits are made and the 
original work is properly cited (including links to both the formal publication through the relevant DOI and the license). See: https://creativecommons.org/licenses/by-nc-nd/4.0/.

\section{References}

1. Mutyaba AK, Ntsekhe M. Tuberculosis and the Heart. Cardiol Clin 2017;35:135-44.

2. Mayosi BM, Burgess LJ, Doubell AF. Tuberculous Pericarditis. Circulation 2005;112:3608-16.

3. Mayosi BM. Contemporary trends in the epidemiology and management of cardiomyopathy and pericarditis in sub-Saharan Africa. Heart (British Cardiac Society) 2007;93:1176-83.

4. Reuter H, Burgess L, van Vuuren W, et al. Diagnosing tuberculous pericarditis. QJM 2006;99:827-39.

5. Noubiap JJ, Agbor VN, Ndoadoumgue AL, et al. Epidemiology of pericardial diseases in Africa: a systematic scoping review. Heart 2019;105:180-8.

6. Mayosi BM, Ntsekhe M, Volmink JA, et al. Interventions for treating tuberculous pericarditis. Cochrane Database Syst Rev 2002;(4):CD000526.

7. Syed FF, Mayosi BM. A Modern Approach to Tuberculous Pericarditis. Prog Cardiovasc Dis 2007;50:218-36.

8. Pandie S, Peter JG, Kerbelker ZS, et al. The diagnostic accuracy of pericardial and urinary lipoarabinomannan (LAM) assays in patients with suspected tuberculous pericarditis. Sci Rep 2016;6:32924.

9. Theron G, Peter J, Calligaro G, et al. Determinants of PCR performance (Xpert MTB/RIF), including bacterial load and inhibition, for TB diagnosis using specimens from different body compartments. Sci Rep 2014;4:5658.

10. Pandie S, Peter JG, Kerbelker ZS, et al. Diagnostic accuracy of quantitative PCR (Xpert MTB/RIF) for tuberculous pericarditis compared to adenosine deaminase and unstimulated interferon- $\gamma$ in a high burden setting: a prospective study. BMC Med 2014;12:101.

11. Zumla A, Maeurer M, Moll G, et al. Host-directed therapies for tuberculous pericarditis. Int J Infect Dis 2015;32:30-1.

12. Ntsekhe M, Mayosi BM. Tuberculous pericarditis with and without HIV. Heart Fail Rev 2013;18:367-73.

13. Syed FF, Sani MU. Recent advances in HIV-associated cardiovascular diseases in Africa. Heart 2013;99:1146-53.

14. Mayosi BM, Ntsekhe M, Bosch J, et al. Prednisolone and Mycobacterium indicus pranii in tuberculous pericarditis. N Engl J Med 2014;371:1121-30.

15. Mutyaba AK, Balkaran S, Cloete R, et al. Constrictive pericarditis requiring pericardiectomy at Groote Schuur Hospital, Cape Town, South Africa: Causes and perioperative outcomes in the HIV era (1990-2012). J Thorac Cardiovasc Surg 2014;148:3058-65.e1.

16. Mnguni AT, Engel ME, Borkum MS, et al. The Effects of Angiotensin Converting Enzyme Inhibitors (ACE-I) on Human N-Acetyl-Seryl-Aspartyl-Lysyl-Proline (AcSDKP) Levels: A Systematic Review and Meta-Analysis. PLoS One 2015;10:e0143338.

17. Mayosi BM, Wiysonge CS, Ntsekhe M, et al. Clinical characteristics and initial management of patients with tuberculous pericarditis in the HIV era: the Investigation of the Management of Pericarditis in Africa (IMPI Africa) registry. BMC Infect Dis 2006;6:2.

18. Xie DL, Cheng B, Sheng Y, et al. Diagnostic accuracy of adenosine deaminase for tuberculous pericarditis: a metaanalysis. Eur Rev Med Pharmacol Sci 2015;19:4411-8.

19. Tuon FF, Litvoc MN, Lopes MI. Adenosine deaminase and tuberculous pericarditis-A systematic review with meta-analysis. Acta Trop 2006;99:67-74.

20. Liu C, Cui YL, Ding CM, et al. Diagnostic accuracy of interferon-gamma in pericardial effusions for tuberculous pericarditis: a meta-analysis. J Thorac Dis 2018;10:854-60.

21. Martinez-Vazquez JM, Ribera E, Ocaña I, et al. Adenosine deaminase activity in tuberculous pericarditis. Thorax 1986;41:888-9.

22. Cegielski JP, Devlin BH, Morris AJ, et al. Comparison of PCR, culture, and histopathology for diagnosis of tuberculous pericarditis. J Clin Microbiol 1997;35:3254-7.

23. Kohli M, Schiller I, Dendukuri N, et al. Xpert ${ }^{\circledR}$ MTB/RIF assay for extrapulmonary tuberculosis and rifampicin resistance. Cochrane Database Syst Rev 2018;8:CD012768.

24. van der Bijl P, Herbst P, Doubell AF. Redefining EffusiveConstrictive Pericarditis with Echocardiography. J Cardiovasc Ultrasound 2016;24:317-23.

25. Chakravorty S, Simmons AM, Rowneki M, et al. The New Xpert MTB/RIF Ultra: Improving Detection of Mycobacterium tuberculosis and Resistance to Rifampin in an Assay Suitable for Point-of-Care Testing. MBio 2017. doi: 10.1128/mBio.00812-17.

26. Adler Y, Charron P, Imazio M, et al. 2015 ESC Guidelines for the diagnosis and management of pericardial diseases: The Task Force for the Diagnosis and Management of Pericardial Diseases of the European Society of Cardiology (ESC)Endorsed by: The European Association for CardioThoracic Surgery (EACTS). Eur Heart J 2015;36:2921-64. 27. Klein AL, Abbara S, Agler DA, et al. American Society 
of Echocardiography clinical recommendations for multimodality cardiovascular imaging of patients with pericardial disease: endorsed by the Society for Cardiovascular Magnetic Resonance and Society of Cardiovascular Computed Tomography. J Am Soc Echocardiogr 2013;26:965-1012.e15.

28. Mulvagh SL, Rokey R, Vick GW 3rd, et al. Usefulness of nuclear magnetic resonance imaging for evaluation of pericardial effusions, and comparison with two-dimensional echocardiography. Am J Cardiol 1989;64:1002-9.

29. Bogaert J, Francone M. Cardiovascular magnetic resonance in pericardial diseases. J Cardiovasc Magn Reson 2009;11:14.

30. Xu B, Harb SC, Klein AL. Utility of multimodality cardiac imaging in disorders of the pericardium. Echo Res Pract 2018. [Epub ahead of print].

31. Dong A, Dong H, Wang Y, et al. (18)F-FDG PET/ CT in differentiating acute tuberculous from idiopathic pericarditis: preliminary study. Clin Nucl Med 2013;38:e160-5.

32. Guidelines for treatment of drug-susceptible tuberculosis and patient care - 2017 update. World Health Organization 2017.

33. Pasipanodya JG, Mubanga M, Ntsekhe M, et al. Tuberculous Pericarditis is Multibacillary and Bacterial Burden Drives High Mortality. EBioMedicine 2015;2:1634-9.

34. Shenje J, Ifeoma Adimora-Nweke F, Ross IL, et al. Poor Penetration of Antibiotics Into Pericardium in Pericardial Tuberculosis. EBioMedicine 2015;2:1640-9.

35. Maisch B, Seferović PM, Ristić AD, et al. Guidelines on the diagnosis and management of pericardial diseases executive summary; The Task force on the diagnosis and management of pericardial diseases of the European society of cardiology. Eur Heart J 2004;25:587-610.

36. Imazio M, Brucato A, Maestroni S, et al. Risk of Constrictive Pericarditis After Acute Pericarditis.

Cite this article as: Naicker K, Ntsekhe M. Tuberculous pericardial disease: a focused update on diagnosis, therapy and prevention of complications. Cardiovasc Diagn Ther 2020;10(2):289-295. doi: 10.21037/cdt.2019.09.20
Circulation 2011;124:1270-5.

37. Damasceno A, Mayosi BM, Sani M, et al. The causes, treatment, and outcome of acute heart failure in 1006 Africans from 9 countries. Arch Intern Med 2012;172:1386-94.

38. Ramasamy V, Mayosi BM, Sturrock ED, et al. Established and novel pathophysiological mechanisms of pericardial injury and constrictive pericarditis. World J Cardiol 2018;10:87-96.

39. Matthews K, Deffur A, Ntsekhe M, et al. A Compartmentalized Profibrotic Immune Response Characterizes Pericardial Tuberculosis, Irrespective of HIV-1 Infection. Am J Respir Crit Care Med 2015;192:1518-21.

40. Ntsekhe M, Matthews K, Syed FF, et al. Prevalence, hemodynamics, and cytokine profile of effusiveconstrictive pericarditis in patients with tuberculous pericardial effusion. PLoS One 2013;8:e77532.

41. Ntsekhe M, Matthews K, Wolske J, et al. Scientific letter: Ac-SDKP (N-acetyl-seryl-aspartyl-lysyl-proline) and Galectin-3 levels in tuberculous pericardial effusion: implications for pathogenesis and prevention of pericardial constriction. Heart 2012;98:1326-8.

42. Ntsekhe M, Shey Wiysonge C, Commerford PJ, et al. The prevalence and outcome of effusive constrictive pericarditis: a systematic review of the literature. Cardiovasc J Afr 2012;23:281-5.

43. Strang JI, Kakaza HH, Gibson DG, et al. Controlled clinical trial of complete open surgical drainage and of prednisolone in treatment of tuberculous pericardial effusion in Transkei. Lancet 1988;2:759-64.

44. Imazio M, Bobbio M, Cecchi E, et al. Colchicine in Addition to Conventional Therapy for Acute Pericarditis. Circulation 2005;112:2012-6.

45. Liebenberg JJ, Dold CJ, Olivier LR. A prospective investigation into the effect of colchicine on tuberculous pericarditis. Cardiovasc J Afr 2016;27:350-5. 